\title{
SPACE BIOSENSOR SYSTEMS: IMPLICATIONS FOR TECHNOLOGY TRANSFER
}

\author{
J. W. Hines*, C. J Somps, and M. Madou \\ NASA - Ames Research Center, MS 213-2, Moffett Field, CA 94035-1000 \\ *E-mail: jhines@mail.arc.nasa.gov
}

\begin{abstract}
To meet the need for continuous, automated monitoring of animal subjects, including humans, during space flight, NASA is developing advanced physiologic sensor and biotelemetry system technologies. The ability to continuously track basic physiological parameters, such as heart rate, blood $\mathrm{pH}$, and body temperature, in untethered subjects in space is a challenging task. At NASA's Ames Research Center, where a key focus is gravitational biology research, engineers have teamed with life scientists to develop wireless sensor systems for automated physiologic monitoring of animal models as small as the rat. This technology is also being adapted, in collaboration with medical professionals, to meet human clinical monitoring needs both in space and on the ground. Thus, these advanced monitoring technologies have important dual-use functions; they meet space flight data collection requirements and constraints, while concurrently addressing a number of monitoring and data acquisition challenges on the ground in areas of clinical monitoring and biomedical research. Additional applications for these and related technologies are being sought and additional partnerships established that enhance development efforts, reduce costs and facilitate technology infusion between the public and private sectors. This paper describes technology transfer and co-development projects that have evolved out of NASA's miniaturized, implantable chemical sensor development efforts.
\end{abstract}

key words: in vivo, chemical sensors, biosensors, biotelemetry, space flight research, technology transfer

\section{INTRODUCTION}

Although humans appear to tolerate increasingly longer tours in space, a number of critical questions remain unanswered about the potential hazards of extended duration space flights from both microgravity and space radiation exposure [1-3]. Understanding the underlying physiological mechanisms associated with space adaptation can help scientists understand the potential hazards and limitations associated with adaptation to various gravitational fields, the feasibility of reproduction and development in microgravity on future outposts, and at a fundamental level, the processes of adaptation and evolution themselves.
As in any scientific investigation, some basic requirements must be met; for example, data must be collected from as many subjects as possible, all other influences must be eliminated, held constant, or at minimum replicated in a control population, and data must be collected at the desired rate and be of highest quality. Sensors and related instrumentation capable of monitoring physical, chemical and biological parameters from unrestrained, small animal subjects are considered "basic" tools for NASA space flight investigators. On earth, however, these same technologies represent significant advancements to existing laboratory and medical facilities. In the pursuit of these critical, yet "basic" space technologies, NASA has discovered a number of dual-use applications and areas of collaboration that are of public benefit in both the short and long run - in the short run by transferring space technologies to home and hospital health care applications and in the long run by enabling better space research to expand the current limits of space exploration.

The Sensors 2000! (S2K!) program at NASA's Ames Research Center in Mountain View, California, provides expertise and facilities for conducting specialized sensor systems research, development, testing and transition to space life sciences research. The Advanced Technology Development-Biosensors (ATD-B) arm acts as a 'technology incubator' to identify new and emerging technologies, then evaluates them in terms of their potential application to future space flight research requirements. The ATD-B project office serves as a valuable national resource by disseminating information about advances in biosensor and biotelemetry technology. It also acts as a liaison for establishing requirements and infusing new technologies between NASA and other entities.

Although several advanced technology development projects are currently underway within the ATD-B program that involve internal and external collaborations with strong technology transfer potential [4], this paper focuses on projects that have evolved out of our miniaturized implantable chemical sensor development efforts.

\section{MINIATURIZED CHEMICAL SENSORS}

In order to meet the needs of the space life sciences research community to continuously monitor various 
chemical and biologic analytes in vivo, the S2K! ATD-B program is developing miniaturized chemical and biosensors suitable for chronic in vivo monitoring. Current efforts are focused on understanding the effects of biologic tissue on the function of polymeric, electruchemical membranes. Polymerbased ion-selective membranes are compatible with miniaturization, can be cast in a variety of sizes and shapes, and have been described extensively in the literature.

\section{A Miniaturized $\mathrm{pH}$ Sensor}

To characterize these membranes, we have developed a simple $\mathrm{pH}$ sensor consisting of an indicator and reference electrode, fabricated from 1.5" lengths of a microbore, nontoxic, medical grade PVC tubing (Fig. 1). The indicator electrode incorporates an $\mathrm{H}^{+}$-selective, neutral carrier (tri-ndodecylamine) doped, PVC membrane cast around the tip of a microbore (0.07" O.D.) PVC catheter. The reference electrode employs a polymerized HEMA (hydroxyethyl methacrylate) plug as a liquid junction frit.

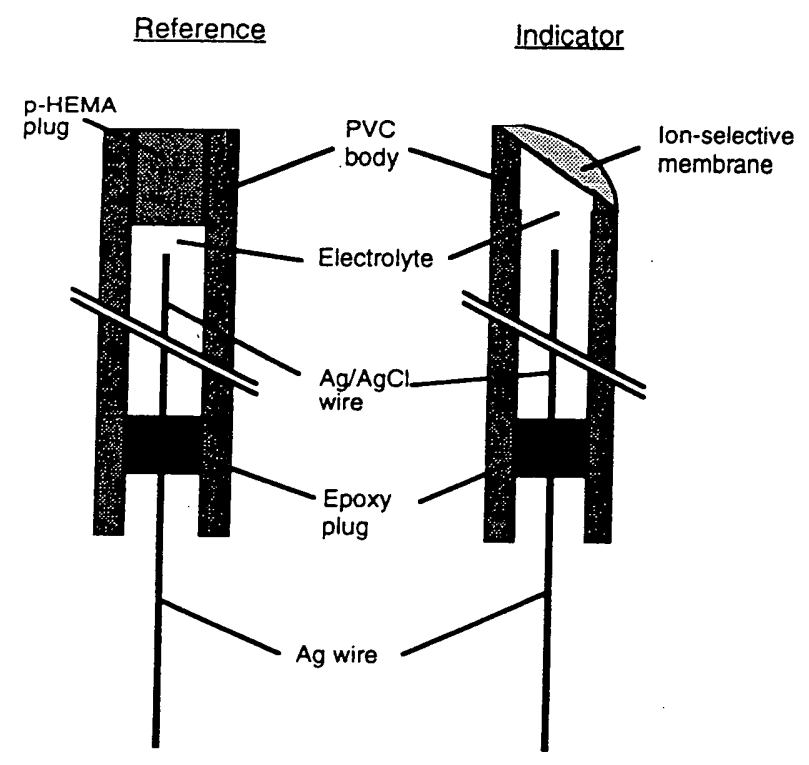

Figure 1. A Miniaturized pH Sensor

These miniaturized $\mathrm{pH}$ sensors exhibit response characteristics similar to larger, commercially available $\mathrm{pH}$ electrodes. Baseline drift is on the order of 0.01-0.03 pH units every 24 hours. Sensitivity is linear between $\mathrm{pH} 4$ and $\mathrm{pH} 9$ and slopes fall between 55 and $59 \mathrm{mV} / \mathrm{pH}$ unit (Nernstian response is $59.16 \mathrm{mV} / \mathrm{pH}$ at $25^{\circ} \mathrm{C}$ ). Sensors are highly selective to $\mathrm{H}^{+}$over other cations (e.g., $10^{11}$ times more selective to $\mathrm{H}^{+}$compared to $\mathrm{Na}^{+}$). Response times are rapid with roughly $95 \%$ of the response achieved within less than 3 seconds.

Extensive testing following implantation in rat subcutaneous tissue has revealed that these sensors retain nearly all of their electrochemical properties following as many as 21 days of implantation [5]. Recent studies indicate this is still true following as many as 56 days of implantation [6]. Actual testing of the sensors in vivo has demonstrated that the sensors are also capable of accurately measuring changes in tissue $\mathrm{pH}$ induced in a rat experiencing mild respiratory acidosis [7].

Chemical sensors are also being integrated with wireless biotelemetry systems to allow long-term evaluation of sensor and membrane performance in animal models, and also to provide technologies to meet NASA's long-term goals for space life sciences research [8].

\section{TECHNOLOGY TRANSFER APPLICATIONS}

Examples of current technology transfer and codevelopment projects that incorporate our miniature implantable, chemical sensor technology and expertise are described below.

\section{UCSF Fetal Monitoring}

NASA sensor and biotelemetry technology is being modified to monitor the human fetus in utero (Fig. 2) [9]. In collaboration with doctors at the University of California at San Francisco Fetal Treatment Center (FTC), NASA's S2K! program is developing a totally implantable biotelemetry system that will monitor the heart rate, body temperature, and tissue $\mathrm{pH}$ of a human fetus following in utero surgery. FTC pediatric surgeons have repaired congenital defects prior to birth in more than 50 cases but are faced with a high incidence of premature birth that carries with it increased mortality and morbidity. Drugs used to prevent premature labor have effects on the infant which could

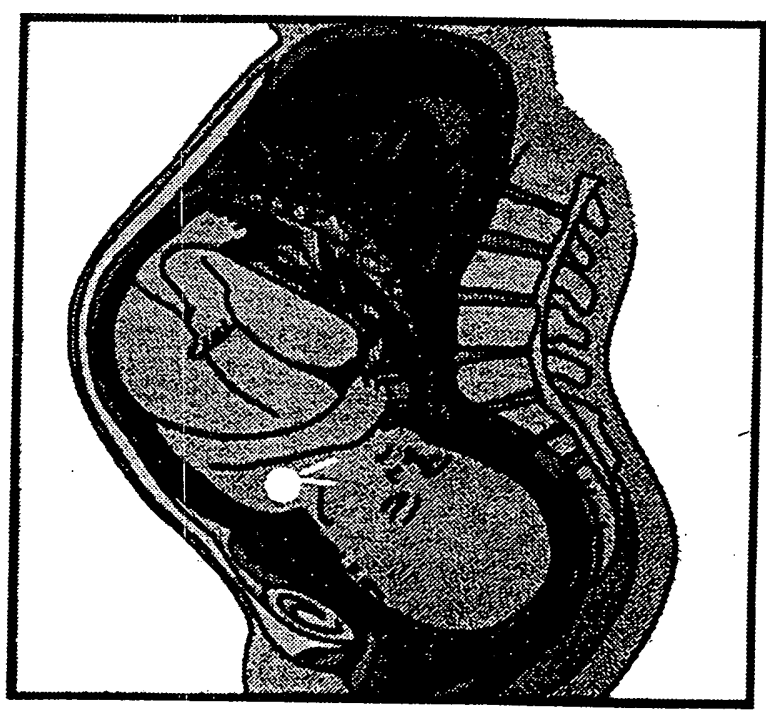

Figure 2. UCSF Fetal Biotelemetry 
be monitored by an on-line $\mathrm{pH}$ sensor in addition to the cardiac monitoring system currently used to titrate medication. The biotelemetry system currently being developed will allow doctors to monitor the infant's general metabolic status directly in utero while treating the mother.

\section{Cedars-Sinai BioArtifical Liver Monitoring}

NASA implantable chemical sensor technology is being incorporated into a device for monitoring the performance of a BioArtificial Liver (BAL) System (Fig 3). In collaboration with doctors at the Cedars-Sinai Medical Center Liver Support Unit (LSU), NASA's S2K! program is developing an artificial liver ion monitoring system that will continuously monitor free calcium and $\mathrm{pH}$ in an ex vivo blood plasma stream during BAL procedures. LSU doctors developed the BAL to "bridge" patients with acute liver failure until a donor liver can be provided. The BAL includes a cell bioreactor loaded with pig liver cells. Blood plasma from the patient is passed through the bioreactor and returned to the patient in a continuous loop. NASA's chemical sensors provide real-time determinations of parameters like $\mathrm{pH}$ and free calcium so that doctors can better control a patient's blood chemistry and thus prolong the duration of effective BAL treatment.

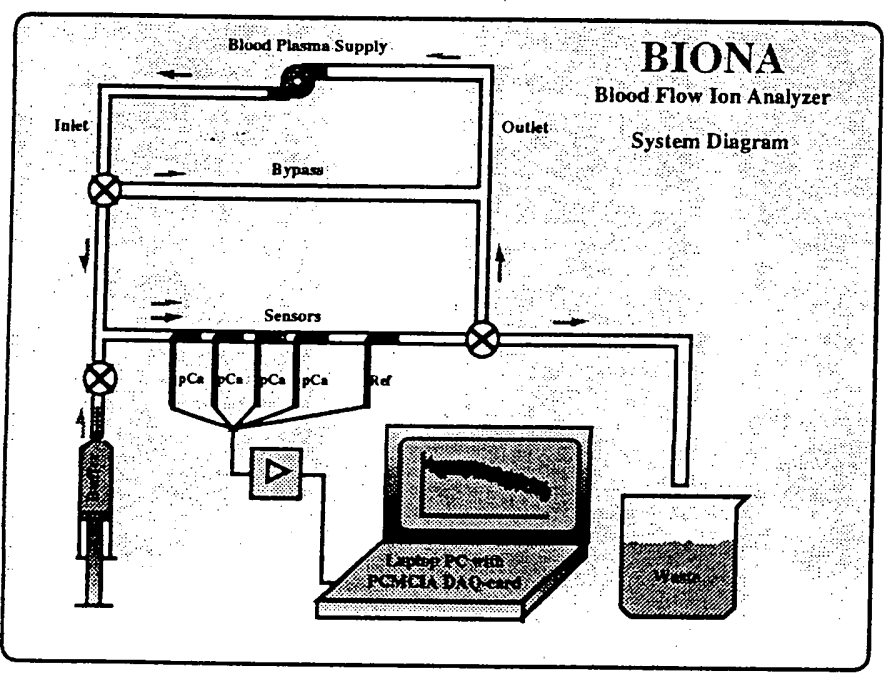

Figure 3. Cedars-Sinai Artifical Liver Monitoring System

\section{Ex Vivo Blood Gas Monitoring}

NASA's S2K! program is working with a small start-up company to develop an ex vivo blood $\mathrm{pH}$ and blood gas monitoring capability. This company is interested in meeting a medical need for a continuous inline, or online, real-time determination of blood chemistry. Toward that end, miniaturized, implantable $\mathrm{pH}$ sensors, developed for space life sciences research, are being adapted for monitoring $\mathrm{pH}$ and $\mathrm{pCO}_{2}$ in whole blood. A miniaturized $\mathrm{pO}_{2}$ sensor is also planned. Current catheter configurations will be used for materials and membrane biocompatibility testing and early proof-of-concept devices, while collaborators at Ohio State University will develop modified electronic circuit fabrication techniques to make the sensors in an inexpensive, easily manufactured, planar configuration.

\section{Cell Culture Monitoring}

Finally, these sensors are being adapted for monitoring analyte concentrations in cell culture media. Cell culture research benefits from close monitoring of both nutrients and waste products. Continuous measurement of parameters such as $\mathrm{pH}$ will allow investigators to optimize cell "feeding" and flushing schedules. The S2K! program is collaborating with investigators at the Walter Reed Army Institute of Research on the development of a small, automated cell culture media monitoring capability based on our miniaturized sensors. Plans to fly these sensors aboard the Space Shuttle as part of a space-based cell culture system in late 1998 are underway.

\section{Additional Projects}

In addition to the sensor-based projects described above, $\mathrm{S} 2 \mathrm{~K}$ ! is actively involved in other technology transfer activities at various stages of maturity. For example, in collaboration with the Ames Commercial Technology Office, a Joint Sponsored Research Agreement was signed between $\mathrm{ARC}$ and a medical information company from Florida. The purpose of the agreement was to apply ATD-B and S2K! developed technologies and expertise in biomedical sensors and biotelemetry instrumentation to remote medical monitoring and home health care systems being developed to interface with the company's information systems network. If successful, the application of these technologies could significantly reduce the costs of caregiving for patients and potentially save lives.

\section{DISCUSSION AND CONCLUSIONS}

Each of the biotechnology development projects described above are the result of spin-off collaborations between NASA and outside partners directly interested in product use. These projects fulfill both space research and earth benefit objectives of NASA. Sensors, biotelemetry and instruments developed within the ATD-B Project are directly targeted to accomplishing the near and far term measurement requirements of specific space flight missions. Additionally, ATD-B has facilitated advanced development and precursor activities in support of Ames' Space Station Biological Research Projects Office sensor and telemetry requirements.

The same technologies are applicable to other groundbased science and space flight programs whose objectives require biomedical and biological data acquisition and monitoring. An active technology development network 
involving other government laboratories, universities, research institutes, commercial and industrial organizations, and specialized technology consultants and vendors has been informally established to support these efforts. Several sensor, biotelemetry, and instrumentation demonstration technologies and products have resulted from these associations.

The ATD-B Project will continue to demonstrate its commitment to coordinate and utilize the skills and expertise of a wide range of resources from the public, private, and academic sectors to produce the most advanced sensor and bioinstrumentation technologies. Via strong partnerships with the medical community and private industry, NASA S2K! will continue to infuse the kind of advanced technologies that the life sciences space research community considers basic to achieving their science objectives, and the ground-based non-NASA community views as next generation improvements to medicine and home health care.

\section{REFERENCES}

[1] A Strategy for Space Biology and Medical Science for the 1980s and 1990s. NRC, Space Science Board. Committee on Space Biology and Medicine, J. Goldberg, Chairman, 1987.

[2] Exploring The Living Universe: A Strategy For Space Life Sciences.- NASA Advisory Council, Life Sciences Strategic Planning Study Committee Report, C. Robbins, Chairman, 1988.

[3] Strategic Considerations For Support Of Humans In - Space And Moon/Mars Exploration Missions. Life Sciences Research And Technology Programs. NASA Advisory Council, Aerospace Medicine Advisory Committee, H. C. Holloway, M.D., Deputy Dean, vols. 1, 2, 1992.

[4] J.W. Hines, "Medical and Surgical Applications of Space Biosensor Technology," Acta Astronautica, vol. 38, pp. 261267, 1996.

[5] C.J. Somps, J.L. Pickering, M.J. Madou, J.W. Hines, D.L. Gibbs, and M.R. Harrison, "Electrochemical performance of an ion selective, polymeric membrane following chronic implantation in rat subcutaneous tissue," Sensors \& Actuators, vol. B35-36, pp. 222-227, 1996.

[6] C.J. Somps M.J. Madou, E. Pickering, and J.W. Hines, "Effects of rat subutaneous tissue on a polymeric membrane $\mathrm{pH}$ sensor," Proceedings of the 14th International Symposium on Biotelemetry, Marburg, Germany, 1997.

[7] S.B. Arnaud, C.J. Somps. A. Chiao, M.J. Madou, and J.W. Hines, "In Vivo Model to Test Implanted Biosensors for Blood pH," Abstract, 68th Annual Scientific Meeting of the Aerospace Medical Association, Chicago, May, 1997.

[8] J.W. Hines, C.J. Somps, M.J. Madou, D.C. Jeutter, and A. Singh, "Telemetric Sensors for the Space Life
Sciences," Proceedings of the IEEE Engineering in Medicine and Biology 18th Annual International Conference, Amsterdam, November, 1996.

[9] J.W. Hines, C.J. Somps, B. Ricks, and L. Kim, "Advanced Biotelemetry Systems for Space Life Sciences: $\mathrm{pH}$ Telemetry," Proceedings of the 13th International Symposium on Biotelemetry, Williamsburg, VA, pp. 131 137, 1995. 